\title{
Análise da composição bioquímica da pele por espectroscopia Raman
}

\author{
Patrícia Karen Oliveira, Maira Gaspar Tosato, Rani de Souza Alves, Airton Abrahão Martin*, \\ Priscila Pereira Fávero, Leandro Raniero
}

Resumo O envelhecimento cutâneo caracteriza-se por alterações celulares e moleculares. Neste contexto, a espectroscopia Raman Confocal foi utilizada para medir estas alterações bioquímicas em função da profundidade da pele in vivo. Neste estudo houve a tentativa de correlacionar os modos vibracionais dos aminoácidos puros com os espectros da pele de voluntárias de diferentes faixas etárias. Foram coletados espectros in vivo de 32 voluntárias, sendo 11 para o Grupo A (20-23 anos), 11 para o Grupo B (39-42 anos) e 10 para o Grupo C (59-62 anos). Para cada grupo, os espectros Raman foram medidos na superfície $(0 \mu \mathrm{m}), 30 \pm 3 \mu \mathrm{m}$ e $60 \pm 3 \mu \mathrm{m}$. Os resultados das comparações intergrupos mostraram que o grupo de maior idade teve prevalência da banda da tirosina, mas também apresentou um decréscimo da banda do ácido pirrolidônico, centrada em $875 \mathrm{~cm}^{-1}$. A banda da amida I, centrada em $1637 \mathrm{~cm}^{-1}$, atribuída à presença de colágeno, como também outras proteínas e lipídeos, apresentou uma menor intensidade no Grupo C, o que pode ser justificado pelo decréscimo na concentração de colágeno em função da idade.

Palavras-chaves Pele humana, Aminoácidos, Espectroscopia Raman.

\section{Skin biochemical composition analysis by Raman spectroscopy}

\begin{abstract}
Skin aging is characterized by cellular and molecular alterations. In this context, Confocal Raman spectroscopy was used in vivo to measure these biochemical changes as function of the skin depth. In this study we have tried to correlate spectra from pure amino acids to in vivo spectra from volunteers with different ages. This study was performed on 32 volunteers: 11 from Group A (20-23 years), 11 from Group B (39-42 years) and 10 from Group C (59-62 years). For each group, the Raman spectra were measured on the surface (0 $\mu \mathrm{m}), 30 \pm 3 \mu \mathrm{m}$ and $60 \pm 3 \mu \mathrm{m}$ below the surface. The results from intergroup comparisons showed that the oldest group had a prevalence of the tyrosine band, but it also presented a decrease in the band centered at $875 \mathrm{~cm}^{-1}$ of pyrrolidone acid. The amide I band centered at $1637 \mathrm{~cm}^{-1}$ that is attributed to collagen, as well as other proteins and lipid, showed a smaller amount of these biomolecules for Group C, which can be explained by the decrease in collagen concentration as a function of age.
\end{abstract}

Keywords Human skin, Amino acids, Raman spectroscopy. 


\section{Extended Abstract}

\section{Introduction}

The skin is composed by epidermis, dermis and hypodermis layers. The water concentration in these layers depend on the natural moisturizing factor (NMF), which is formed mainly by the pyrrolidone carboxylic acid (PCA), urocanic acid, lactate, urea, serine, glycine, arginine, ornithine, citrulline, alanine, histidine and phenylalanine (Caspers et al., 2003; Harding et al., 2000). The NMF is essential to maintain the stratum corneum mechanical properties. However, measuring these compounds concentration without changing in the physical and chemical properties of the skin is still a complicated procedure.

In this context, dermatologists, cosmetic companies and researchers have studied non-invasive alternative to evaluate the biochemical composition of the skin structures. Recently Confocal Raman spectroscopy has been used in the characterization of biological tissues in vivo at different depths (Egawa e Tagami, 2007). This technique is based on interaction of biological tissue with a laser beam. The light scattered by the sample has information about the chemical bonds and it may determine the particular molecular group amount. These data can be monitored in real time without sample degradation. In fact, Raman spectroscopy is a powerful tool, being used both for diagnosis of disease, either in vivo (Raniero et al., 2011) as well as the analysis of body fluids (Carvalho et al., 2011).

In this paper, the main NMF amino acids were analyzed separately by Raman spectroscopy. Then, an attempt was made to correlate them with the skin spectra of women measured at different ages and skin depths.

\section{Material and Methods}

This study was approved by the Ethics Committee in Research of the Universidade do Vale do Paraiba following the Guidelines and Norms Regulating Research Involving Human Subjects, under the National Health Protocol H48/CEP/2008. The study was developed with 32 volunteers, 11 from Group A (20-23 years), 11 from group $B$ (39-42 years) and 10 from Group $C$ (59-62 years). This work also involves different skin phototypes according to Fitzpatrick classification.

All data were analyzed at the Laboratory of Biomedical Vibrational Spectroscopy.

The analyzed amino acids were the pyrrolidone carboxylic acid, arginine, ornithine, citrulline, glycine, serine, histidine, proline and phenylalanine, which was purchased from Sigma-Aldrich and measured by Raman spectroscopy. It was used a $785 \mathrm{~nm}$ diode laser (Sacher LASER Technik) and the power in the samples was $25 \mathrm{~mW}$. The spectrometer was Spectra Pro $2500 \mathrm{i}$ of PiActon ${ }^{\circledR}$, with a diffraction grating of 300 grooves $/ \mathrm{mm}$ coupled in a CCD camera refresh by liquid nitrogen and the spectral resolution was $7 \mathrm{~cm}^{-1}$.

The skin spectra were performed using a Confocal Raman probe from Jobin Yvon, with a $50 X$ objective and a $785 \mathrm{~nm}$ frequency-stabilized laser. The depth profile was controlled by an translation stage with an accuracy of $\pm 3 \mu \mathrm{m}$. Thorlabs, model LNR50SE. The power at skin was kept at $27 \mathrm{~mW}$ with an integration time of 120 seconds. The spectra were collected after proper cleaning of the skin.

\section{Discussion and Conclusions}

The results showed that Confocal Raman spectroscopy gives the information about the skin depth profile and it helps the understanding of aging process. Results from intergroup comparisons showed that the oldest group had a prevalence of the tyrosine band, but it also presented a decrease in the band centered at $875 \mathrm{~cm}^{-1}$ from pyrrolidone acid. The amide I band centered at $1637 \mathrm{~cm}^{-1}$ that is attributed to collagen, as well as other proteins and lipid, showed a smaller amount of these biomolecules for Group C, which can be explained by the decrease in collagen concentration as a function of age. However, the complexity of the skin Raman spectrum showed that the correlation between the Raman spectra of pure amino acid is not directly obtained. 


\section{Introdução}

A pele é composta pelas camadas da epiderme, derme e hipoderme. A concentração de água nestas camadas depende do fator natural de hidratação (NMF - Natural moisturizer factor), que é formado, principalmente, por ácido carboxílico da pirrolidona (PCA), ácido urocânico, lactato, uréia, serina, glicina, arginina, ornitina, citrulina, alanina, histidina e fenilalanina (Caspers et al., 2003; Harding et al., 2000). O NMF é indispensável para manter as propriedades mecânicas do estrato córneo, pois tem uma ação lipofilmógena. Entretanto, medir a concentração deste composto sem alterar as propriedades físicas e químicas da pele ainda é um procedimento complexo.

Neste contexto, dermatologistas, pesquisadores e empresas cosméticas têm estudado alternativas não invasivas na avaliação da composição bioquímica das estruturas da pele. Recentemente, a espectroscopia Raman Confocal tem sido utilizada na caracterização de tecidos biológicos in vivo em diferentes profundidades (Egawa e Tagami, 2007). Esta técnica é baseada na irradiação do tecido biológico por um laser. A energia espalhada pela amostra tem informações sobre as ligações químicas, podendo determinar o aumento ou decréscimo da quantidade de um dado grupo molecular. Estes dados podem ser monitorados em tempo real, sem nenhuma degradação da amostra. De fato, a espectroscopia Raman tem mostrado ser uma ferramenta bastante versátil, sendo utilizada tanto no diagnóstico de doenças, quer seja in vivo (Raniero et al., 2011), como também na análise de fluídos corporais (Carvalho et al., 2011).

No presente trabalho, os principais aminoácidos que constituem o NMF foram analisados separadamente por Espectroscopia Raman. Então, foi feita uma tentativa de correlacioná-los com os espectros da pele de mulheres de diferentes faixas etárias e diferentes profundidades.

Tal caracterização do maior tecido do corpo humano visa à contribuição do ponto de vista da ciência fundamental, bem como a aplicações tecnológicas através de informações a serem utilizadas pela indústria cosmecêutica.

\section{Materiais e Métodos}

Este trabalho foi desenvolvido no Laboratório de Espectroscopia Vibracional Biomédica no Instituto de Pesquisa e Desenvolvimento da Universidade do Vale do Paraíba, após a aprovação pelo Comitê de Ética em Pesquisa de tal instituição, seguindo as Diretrizes e Normas Regulamentadoras de Pesquisa Envolvendo Seres Humanos, conforme Resolução n ${ }^{\text {o } 196 / 96}$ do Conselho Nacional de Saúde sob o protocolo $\mathrm{n}^{\circ} \mathrm{H} 48 / \mathrm{CEP} / 2008$.

\section{Medidas dos aminoácidos}

Os aminoácidos que compõem o NMF, sendo eles, o ácido carboxílico da pirrolidona, a arginina, a ornitina, a citrulina, a glicina, a serina, a histidina, a prolina e a fenilalanina foram adquiridos pela empresa Sigma-Aldrich e medidos pela técnica de espectroscopia Raman. A fonte de excitação foi um laser com comprimento de onda de $785 \mathrm{~nm}$ (Sacher LASER Technik) e potência na amostra de $25 \mathrm{~mW}$. O espectrômetro utilizado foi o Spectra Pro 2500i da PiActon, com grade de difração de 300 ranhuras $/ \mathrm{mm}$, que foi acoplado a uma câmera CCD resfriada por nitrogênio líquido. A resolução espectral foi de $7 \mathrm{~cm}^{-1}$.

Cada aminoácido foi colocado em um porta-amostra, onde duas medidas foram feitas em pontos diferentes e, posteriormente, foi calculada a média espectral. O tempo total de integração foi igual a 120 segundos, divididos em 2 acumulações de 60 segundos em cada ponto da amostra, com exceção do ácido carboxílico da pirrolidona, o qual teve tempo de 20 segundos e 2 acumulações para cada um dos dois pontos. Após a obtenção dos espectros, foi realizada a subtração da fluorescência utilizando um algoritmo do software Matlab 6.1 ${ }^{\circledR}$. Nesta rotina matemática, pode-se determinar o grau do polinômio e a faixa espectral de interesse, possibilitando o ajuste da melhor linha de base. O melhor ajuste desta linha de base foi feito com polinômio de grau 6 e a região espectral escolhida corresponde aos modos vibracionais de impressão digital para o tecido biológico analisado, ou seja, foi de $750 \mathrm{~cm}^{-1}$ a $1800 \mathrm{~cm}^{-1}$. Após a subtração da fluorescência, realizou-se a normalização e o cálculo da média dos espectros com o auxílio do programa Origin $7.5^{\circledR}$.

\section{Processo de seleção das voluntárias}

O estudo foi composto por 32 voluntárias, as quais foram separadas em três grupos, sendo 11 para o Grupo A (20 a 23 anos), 11 para o grupo B (39 a 42 anos) e 10 para o Grupo C (59 a 62 anos). Buscou-se neste trabalho envolver o maior número possível de diferentes fototipos de pele segundo a classificação de Fitzpatrick (Tabela 1). Essa classificação atribui a numeração de I a VI de acordo com a resposta da pele à exposição ao sol, partindo da pele mais sensível ao tipo de pele com maior tolerância à radiação solar.

As voluntárias foram selecionadas, segundo os critérios de exclusão: histórico em algum momento da vida de dermatoses averiguadas por um dermatologista, de irritação ou sensibilidade a qualquer produto cosmecêutico ou de qualquer tipo de tratamento intensivo relacionado à pele. Além disso, foram 
excluídas voluntárias que faziam uso de corticoides, ou que teriam necessidade de qualquer alteração medicamentosa durante o período de estudo e grávidas e/ou lactantes.

\section{Metodologia experimental in vivo}

As avaliações da pele foram realizadas utilizando uma sonda Raman Confocal, marca Jobin Yvon, equipada com uma objetiva de 50X, um laser de excitação com comprimento de onda de $785 \mathrm{~nm}$ e um controlador de profundidade com precisão de $\pm 3 \mu \mathrm{m}$, marca Thorlabs,

Tabela 1. Classificação dos fototipos de pele por Fitzpatrick (Guirro e Guirro, 2002).

Table 1. Fitzpatrick's skin phototypes (Guirro and Guirro, 2002).

\begin{tabular}{ccc}
\hline $\begin{array}{c}\text { Tipo da } \\
\text { pele }\end{array}$ & Reação & Cor da pele \\
\hline I & Sempre queima, nunca bronzeia & Clara \\
II & Sempre queima, bronzeia pouco & Clara \\
III & Queima e bronzeia pouco & Clara \\
IV & $\begin{array}{c}\text { Raramente queima, bronzeia } \\
\text { com facilidade }\end{array}$ & Morena-clara \\
& $\begin{array}{c}\text { Queima muito raramente, } \\
\text { bronzeia facilmente }\end{array}$ & Morena \\
V & Nunca queima, bronzeia & Negra \\
VI & facilmente &
\end{tabular}

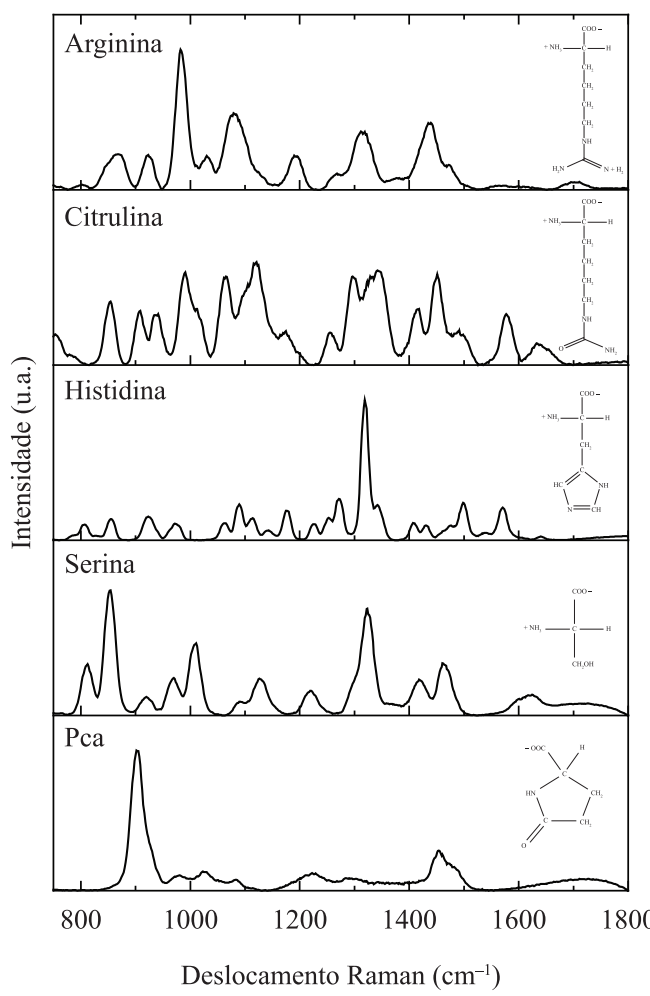

modelo LNR50SE. A potência de irradiação na pele foi de, aproximadamente, $27 \mathrm{~mW}$ com tempo total de integração igual a 120 segundos. A potência do laser foi ajustada de forma que os sinais fossem obtidos com a melhor razão sinal/ruído sem que houvesse aquecimento da amostra ou quaisquer danos à pele da voluntária. Antes de iniciar as avaliações da pele, as voluntárias permaneceram 15 minutos em ambiente climatizado a $23{ }^{\circ} \mathrm{C}$ e com umidade relativa de, aproximadamente, $51 \%$. As análises foram realizadas na região inferior do antebraço após a assepsia do local com algodão embebido em 1,0 mL de álcool etílico $97 \%$. Foram coletados 3 espectros em 3 profundidades distintas: superfície, $30 \pm 3 \mu \mathrm{m}$ e $60 \pm 3 \mu \mathrm{m}$ abaixo da superfície da pele. A fluorescência foi subtraída por ajuste de curva polinomial de grau 6 e os espectros resultantes foram normalizados em relação ao modo vibracional do grupo funcional em $1445 \mathrm{~cm}^{-1}$, de proteínas e lipídeos.

\section{Resultados}

Na Figura 1 têm-se os espectros Raman dos principais constituintes do NMF da pele. Os espectros das substâncias puras fornecem padrões de comparação,

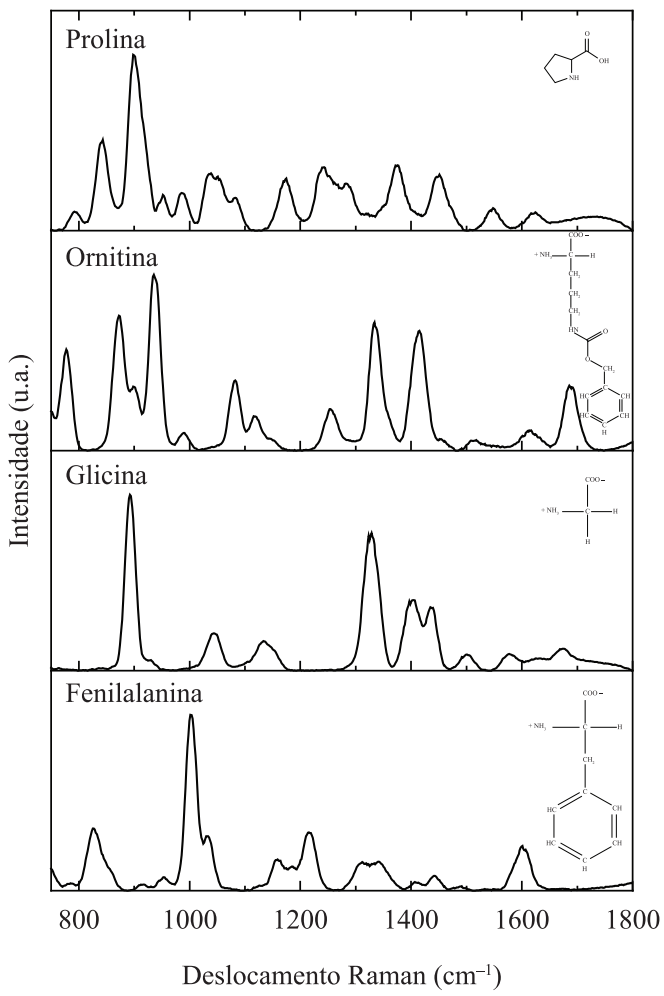

Figura 1. Espectros Raman dos principais constituintes do NMF da pele. Figure 1. Raman spectra of the main constituents of NMF skin. 
que possibilitam identificar as influências destes modos vibracionais nos espectros Raman da pele.

Entretanto, neste trabalho foi analisada apenas uma banda dos modos vibracionais do ácido carboxílico da pirrolidona (PCA), sendo este, um dos principais componentes do NMF (Caspers et al., 2003). Paralelamente, estudou-se uma banda dos modos vibracionais da prolina visto que esta exerce forte influência na constituição do colágeno (Phillips et al., 1994). Com a análise desses constituintes, foi possível

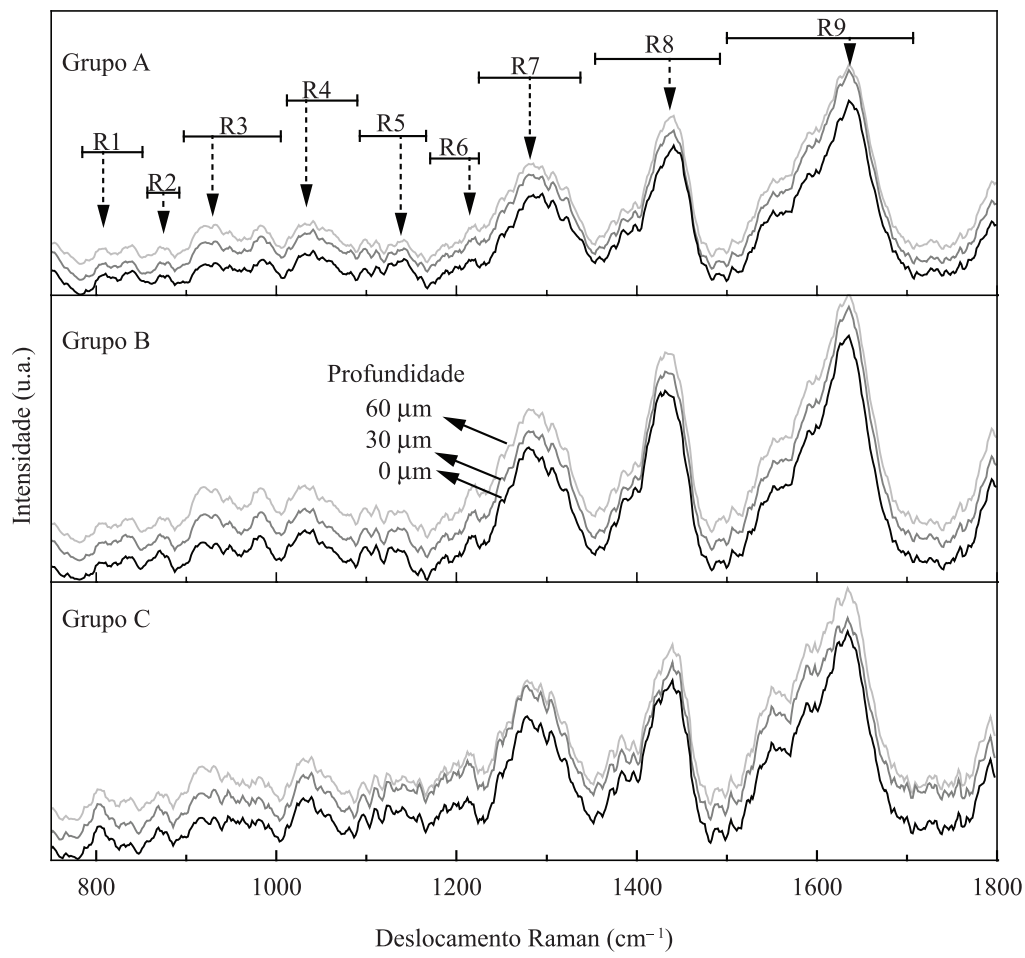

Figura 2. Espectros Raman das médias espectrais dos grupos A, B e C, medidas em diferentes profundidades: superfície $(0 \mu \mathrm{m}), 30 \pm 3 \mu \mathrm{m}$ e $60 \pm 3 \mu \mathrm{m}$ abaixo da superfície da pele.

Figure 2. Raman mean spectra of groups A, B and C, measured at different depths: the skin surface $(0 \mu \mathrm{m}), 30 \pm 3 \mu \mathrm{m}$ and $60 \pm 3 \mu \mathrm{m}$ below the skin surface.

Tabela 2. Atribuições dos modos vibracionais de espectros Raman da pele, onde $\mathrm{v}=$ estiramento e $\delta=$ deformação. Os valores das bandas atribuídas neste trabalho foram feitas com uma revisão da literatura (Caspers et al., 1998; Penteado et al., 2008; Tfayli et al., 2007).

Table 2. Raman assignments of major vibrational modes for the human skin, where $v=$ stretch and $\delta=$ deformation. Values and assignments used in this work were defined based on other literature reports (Caspers et al., 1998; Penteado et al., 2008; Tfayli et al., 2007).

\begin{tabular}{ccc}
\hline Posição $\left(\mathbf{c m}^{-1}\right)$ & Estrutura & Atribuição \\
\hline 808 & $\delta(\mathrm{CCH})$ alifático & Tirosina \\
839 & $\delta(\mathrm{CCH})$ aromático & Tirosina \\
874 & $\mathrm{v}(\mathrm{CC}) ; \mathrm{v}(\mathrm{CN}), \mathrm{PCA}$ & Ác. Pirrolidônico \\
930 & $\mathrm{v}(\mathrm{CC}) \alpha$-hélice & Carotenoides e colesterol \\
985 & $\delta(\mathrm{CCH})$ & Oleofinas \\
1040 & $\mathrm{v}(\mathrm{CC})$ cadeia randômica & Lipídeos \\
1141 & $\mathrm{v}(\mathrm{CC})$ cadeia trans & Lipídeos \\
1217 & ------ & Tirosina e fenilalanina \\
1249 & $\delta(\mathrm{CH})$ wag; $\delta(\mathrm{CN})$ & Amida III desordenada \\
1283 & $\mathrm{v}(\mathrm{CN}), \delta(\mathrm{NH})$ & Amida III $\alpha$-hélice \\
1293 & $\delta(\mathrm{CH})$ & ------ \\
1398 & $\delta(\mathrm{CH})$ & NMF \\
1440 & $\delta(\mathrm{CH})$ & Lipídeos e proteínas \\
1557 & $\mathrm{v}(\mathrm{C}=\mathrm{C})$ & Oleofinas \\
1591 & $\mathrm{v}(\mathrm{C}=\mathrm{C})$ & Oleofinas, tirosina e fenilalanina \\
1636 & $\mathrm{v}(\mathrm{C}=\mathrm{O})$ & Amida I $\alpha$-hélice \\
\hline
\end{tabular}


compreender melhor o processo do envelhecimento cutâneo em diferentes faixas etárias e profundidades da pele. A Tabela 2 contem os principais modos vibracionais da pele com suas estruturas e atribuições.

$\mathrm{Na}$ Figura 2 veem-se os espectros Raman médios da superfície da pele $(0 \mu \mathrm{m})$ e das profundidades $30 \pm 3 \mu \mathrm{m}$ e $60 \pm 3 \mu \mathrm{m}$ dos grupos A, B e C. As faixas espectrais indicadas de R1 a R9 são as regiões escolhidas para o cálculo das áreas, onde as setas indicam as bandas dos modos vibracionais de maior interesse.

As áreas foram calculadas para as bandas que correspondem às biomoléculas com diversas funções

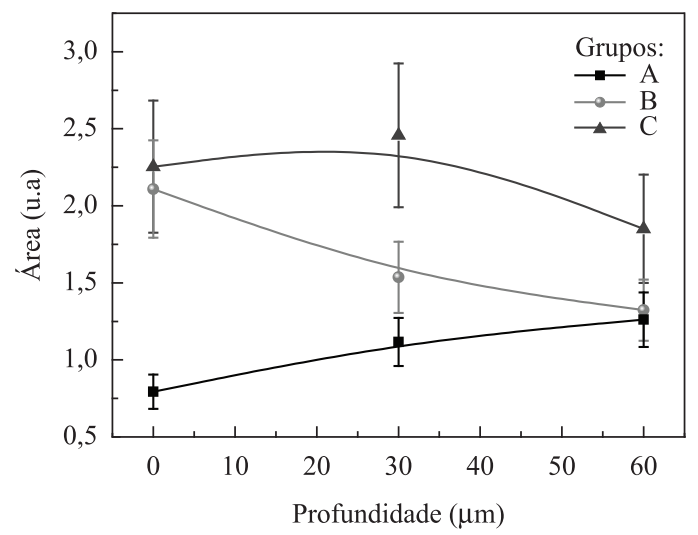

Figura 3. Áreas calculadas pela integração das bandas dos modos vibracionais na região de 849 a $887 \mathrm{~cm}^{-1}(\mathrm{R} 2)$, dos grupos $\mathrm{A}, \mathrm{B}$ e C. As linhas mostram as tendências em função da profundidade.

Figure 3. Areas of bands, of the three groups $(A, B$ and $C)$, resulting from curve fitting of Raman spectra in the frequency range of 849 to $887 \mathrm{~cm}^{-1}(\mathrm{R} 2)$. The lines show the tendency of the areas depending on the depth.

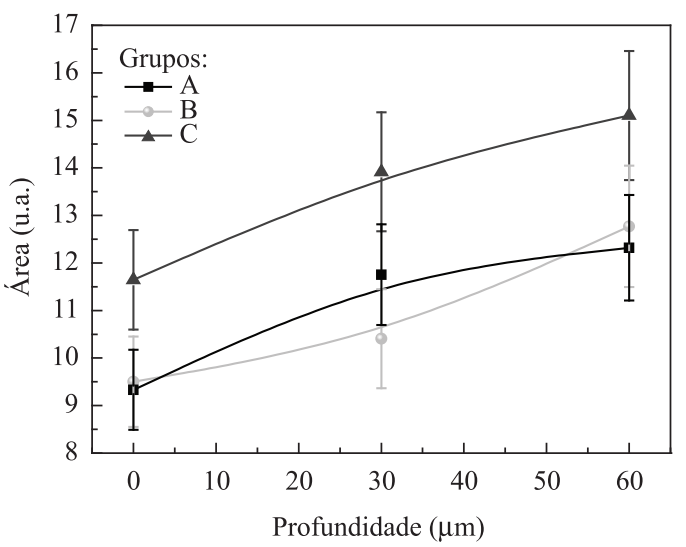

Figura 4. Áreas calculadas pela integração das bandas dos modos vibracionais na região de 887 a $1005 \mathrm{~cm}^{-1}(\mathrm{R} 3)$, dos grupos A, B e C. As linhas mostram as tendências em função da profundidade. Figure 4. Areas of bands, of the three groups ( $A, B$ and $C)$, resulting from curve fitting of Raman spectra in the frequency range of 887 to $1005 \mathrm{~cm}^{-1}$ (R3). The lines show the tendency of the areas depending on the depth. na pele. Os cálculos de área fornecem informações sobre o aumento ou a diminuição na quantidade destas distintas biomoléculas da pele. Os resultados dos cálculos de área estão expressos, sob a forma de gráficos, nas Figuras de 3 a 8 para uma melhor visualização e discussão dos dados encontrados. Em todos os gráficos, os desvios padrão estão indicados pelas barras e os valores médios das áreas estão indicados pelos pontos.

Os valores de área calculados para a região entre 849 e $887 \mathrm{~cm}^{-1}$ (R2), para os três grupos nas três profundidades estudadas podem ser visualizados na Figura 3.

Na Figura 4 podem ser vistos os valores de área calculados na região entre 887 e $1005 \mathrm{~cm}^{-1}$ (R3) para as três profundidades dos três grupos estudados. Os grupos mostraram aumentos aproximadamente lineares, crescentes de valor de área em função do aumento da profundidade.

$\mathrm{Na}$ Figura 5 encontra-se a dependência dos valores das áreas calculadas para a região de 1168 a $1226 \mathrm{~cm}^{-1}$ (R6) em função da profundidade da pele e da idade das voluntárias. Neste gráfico, verificou-se a semelhança dos valores medidos nos grupos A e B, que apresentam uma leve tendência de decréscimo com o aumento da profundidade.

Os valores de área calculados, na região entre 1226 e $1355 \mathrm{~cm}^{-1}$ (R7), centrada em $1283 \mathrm{~cm}^{-1}$ são encontrados na Figura 6. Ao avaliar as funções relativas de cada grupo e as respectivas barras de erro, verificaram-se diferenças significativas das áreas calculadas na superfície da pele, convergindo com o aumento da profundidade.

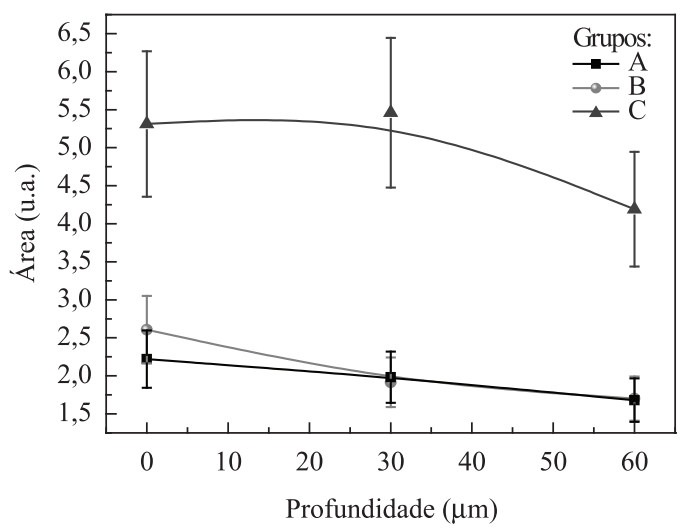

Figura 5. Áreas calculadas pela integração das bandas dos modos vibracionais na região de 1168 a $1226 \mathrm{~cm}^{-1}$ (R6), dos grupos A, B e C. As linhas mostram as tendências em função da profundidade. Figure 5. Areas of bands, of the three groups ( $A, B$ and $C$ ), resulting from curve fitting of Raman spectra in the frequency range of 1168 to $1226 \mathrm{~cm}^{-1}$ (R6). The lines show the tendency of the areas depending on the depth. 
Observam-se na Figura 7 os valores de área calculados, na região de 1355 a $1483 \mathrm{~cm}^{-1}$ (R8). Nessa faixa, a banda dos modos vibracionais levada em consideração foi a centrada em $1441 \mathrm{~cm}^{-1}$ atribuída à presença de lipídeos e proteínas. A análise das barras de erro das medidas nos três grupos de estudo e as diferenças percentuais de aproximadamente $10 \%$ de $C$ em relação a A e B, permitiram a avaliação de que os três grupos apresentaram um comportamento funcional muito semelhante da quantidade de lipídeos e proteínas em relação à profundidade. Assim, pode-se inferir que não foi verificada uma alteração significativa da quantidade de lipídeos e proteínas em relação à idade e em relação à profundidade.

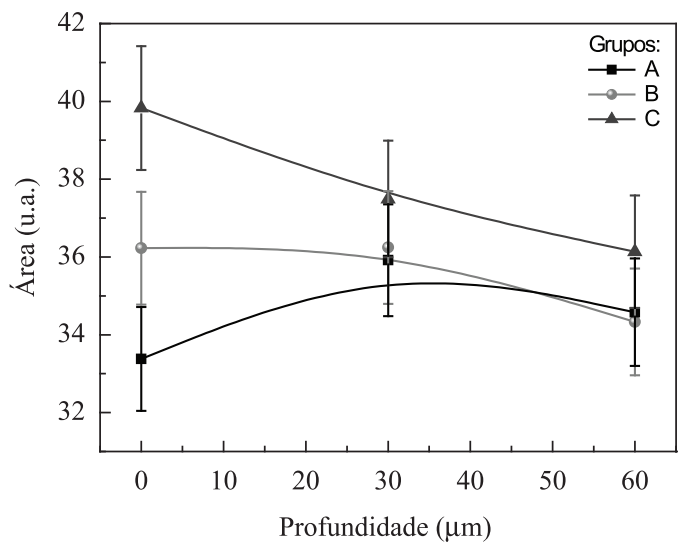

Figura 6. Áreas calculadas pela integração das bandas dos modos vibracionais na região de 1226 a $1355 \mathrm{~cm}^{-1}$ (R7), dos grupos A, B e C. As linhas mostram as tendências em função da profundidade. Figure 6. Areas of bands, of the three groups ( $A, B$ and $C)$, resulting from curve fitting of Raman spectra in the frequency range of 1226 to $1355 \mathrm{~cm}^{-1}(R 7)$. The lines show the tendency of the areas depending on the depth.

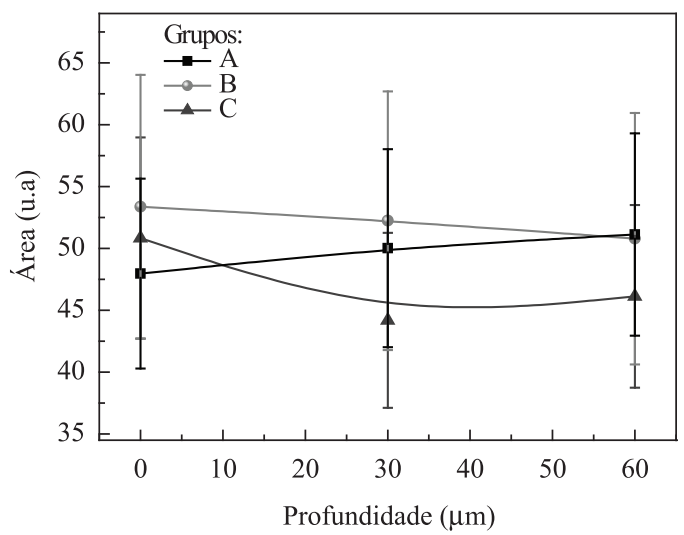

Figura 7. Áreas calculadas pela integração das bandas dos modos vibracionais na região de 1355 a $1483 \mathrm{~cm}^{-1}$ (R8), dos grupos A, B e C. As linhas mostram as tendências em função da profundidade.

Figure 7. Areas of bands, of the three groups $(A, B$ e C), resulting from curve fitting of Raman spectra in the frequency range of 1355 to $1483 \mathrm{~cm}^{-1}$ (R8). The lines show the tendency of the areas depending on the depth.
Os valores de área calculados, na região de 1483 a $1708 \mathrm{~cm}^{-1}$ (R9), para os três grupos nas três profundidades estudadas são encontrados na Figura 8. Nessa faixa, a banda dos modos vibracionais levada em consideração foi a centrada $1637 \mathrm{~cm}^{-1}$, atribuída à presença de amida I. As barras de erro dos três grupos e as diferenças percentuais de aproximadamente $10 \%$ entre o máximo e o mínimo valor de área, mostram uma tendência crescente do grupo funcional amida com a profundidade da pele. O grupo mais jovem apresentou uma quantidade de amida maior que os demais, com um ponto máximo em $30 \pm 3 \mu \mathrm{m}$.

\section{Discussão}

De acordo com a Tabela 2, as bandas dos modos vibracionais centradas aproximadamente em 808 , $839,1217,1591 \mathrm{~cm}^{-1}$, correspondem à presença de tirosina na pele, que é responsável pela síntese de melanina. A presença da enzima tirosinase, concentrada no aparelho de golgi dos melanócitos é responsável pela produção de tirosina. O pigmento é originado a partir da polimerização do aminoácido tirosina por intermédio da ação da tirosinase, a qual passa de aminoácido incolor a um pigmento castanho (Oliveira et al., 2004).

A tirosina polimerizada deposita-se em vesículas denominadas melanossomas, as quais se deslocam pelos prolongamentos citoplasmáticos dos melanócitos e são então transferidas para os queratinócitos através de um processo de secreção denominado secreção citócrina (de célula para célula). Os grânulos de melanina permanecem no citoplasma dos queratinócitos (Oliveira et al., 2004).

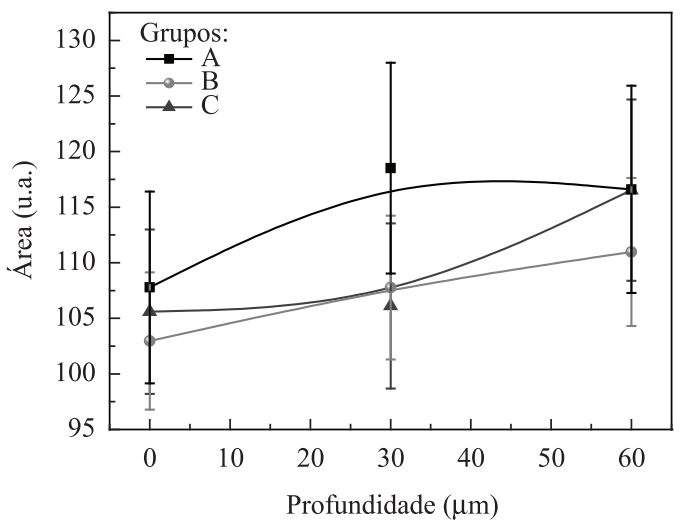

Figura 8. Áreas calculadas pela integração das bandas dos modos vibracionais na região de 1483 a $1708 \mathrm{~cm}^{-1}$ (R9), dos grupos A, B e C. As linhas mostram as tendências em função da profundidade. Figure 8. Areas of bands, of the three groups ( $A, B$ and $C)$, resulting from curve fitting of Raman spectra in the frequency range of 1483 to $1708 \mathrm{~cm}^{-1}$ (R9). The lines show the tendency of the areas depending on the depth. 
A banda dos modos vibracionais centrada em $874 \mathrm{~cm}^{-1}$ refere-se à presença de ácido pirrolidônico, que é um dos principais constituintes do NMF da pele (Caspers et al., 1998; Penteado et al., 2008; Tfayli et al., 2007). O PCA e seus derivados são estudados em virtude da capacidade de hidratar o estrato córneo e são bastante utilizados em produtos cosmecêuticos com a função de melhorar a pele seca e escamosa (Walters e Roberts, 2008).

Em $930 \mathrm{~cm}^{-1}$ é atribuída a presença de carotenoides na pele. Lademann et al. (2009) mostraram um método não invasivo para a determinação in vivo de carotenoides na pele humana e observaram que os indivíduos com altos níveis desses constituintes têm menos sulcos e rugas que indivíduos com menores níveis de antioxidantes. Porém, os carotenoides não podem ser produzidos pelo organismo e precisam ser incorporados na dieta rica em frutas e legumes.

Já em 985, 1040 e $1141 \mathrm{~cm}^{-1}$ atribuem-se à presença de lipídeos e colesterol na pele, os quais exercem a importante função de barreira no estrato córneo, cujas integridade e organização são fundamentais para prevenir a aumentada perda de água transepidermal (Caspers et al., 1998; Penteado et al., 2008; Tfayli et al., 2007).

A amida III, relacionada ao precursor do colágeno, pró-colágeno, apresenta as bandas vibracionais centradas em 1249, 1283 e $1293 \mathrm{~cm}^{-1}$ (Caspers et al., 1998; Penteado et al., 2008; Tfayli et al., 2007).

A banda centrada em $1398 \mathrm{~cm}^{-1}$ relaciona-se aos modos vibracionais do NMF, o qual é composto por moléculas de baixo peso molecular, higroscópicas que previnem assim, a sua evaporação. Essas moléculas são, principalmente, aminoácidos, ácido pirrolidônico carboxílico, lactato de sódio, ácido lático e ureia (Caspers et al., 1998; Penteado et al., 2008; Tfayli et al., 2007).

A banda dos modos vibracionais centrada em $1440 \mathrm{~cm}^{-1}$ refere-se à presença de lipídeos e proteínas e apresentam como função impermeabilizar a pele, prevenindo a perda de água (Caspers et al., 1998; Penteado et al., 2008; Tfayli et al., 2007).

Em 1557 e $1591 \mathrm{~cm}^{-1}$ constatam-se as presenças de oleofinas, fenilalanina e tirosina, respectivamente (Caspers et al., 1998; Penteado et al., 2008; Tfayli et al., 2007).

E, por último, a banda dos modos vibracionais, centrada em $1636 \mathrm{~cm}^{-1}$, é atribuída à presença de amida I, relativa ao colágeno (Movasaghi et al., 2007). Os modos vibracionais característicos da amida I são estiramento $\mathrm{C}=\mathrm{O}$, com menor contribuição de deformação de N-H (Choi e Cho, 2009).

Nas Figuras 3 e 4 as regiões das bandas vibracionais atribuídas ao ácido pirrolidônico puro, como também às presenças de colesterol e carotenoides, foram correlacionadas com a pele. Entretanto, observa-se que o grupo $\mathrm{C}$ tem os maiores valores de área relativa que os demais, contrariando outros estudos (Berry et al., 1999; Engelke et al., 1997; Harding et al., 2000). Com o aumento da idade, a pele torna-se mais seca e os níveis de NMF diminuem em comparação com a de jovens, refletindo, principalmente, em uma redução natural devido à baixa síntese de profilagrina (Harding et al., 2003). Esta discordância entre os resultados pode ser explicada pela contribuição mais acentuada nesta região de outros grupos funcionais, como por exemplo, a tirosina.

$\mathrm{Na}$ Figura 5, os maiores valores das áreas para o grupo C podem ser explicados, pois a tirosina é metabolicamente sintetizada a partir da fenilalanina e contem um anel aromático ligado a um grupo hidroxila, o que a faz mais hidrofílica do que a fenilalanina. Este aminoácido com a hidroxila participa de diversas sínteses importantes, dentre elas o pigmento da melanina. Com o aumento da idade ocorre maior concentração desse pigmento levando ao aparecimento de manchas indesejadas na pele senil (Nicoletti et al., 2002). Este resultado também reforça a hipótese da tirosina ter influenciado os resultados mostrados nas Figuras 3 e 4, pois com a idade existe um decréscimo do PCA, mas um acréscimo da tirosina. Como ambos possuem modos vibracionais nestas regiões, há uma competição, o que dificulta o entendimento dos efeitos do envelhecimento na produção de certas biomoléculas.

Na Figura 6 observa-se que a diferença dos valores das áreas de $\mathrm{C}$ com relação às áreas de $\mathrm{A}$ e $\mathrm{B}$ são pequenas, onde se pode notar que o maior valor encontrado da área sob a curva foi para o grupo $\mathrm{C}$ para a superfície. Este comportamento está ligado à presença do grupo amino secundário de resíduos de prolina constituído em uma conformação rígida, que reduz estruturalmente a flexibilidade das regiões contendo prolina (Nelson e Cox, 2008). Assim, o maior valor de área sob a curva para o grupo $\mathrm{C}$, composto de indivíduos com maior faixa etária, é associado às alterações do processo do envelhecimento que atinge este grupo. A pele do idoso é estrutural e funcionalmente diferente de outros grupos etários. A epiderme é fina e tem a renovação celular mais lenta, resultando em menos resistência ao dano externo (Laube, 2004).

$\mathrm{Na}$ Figura 7, as áreas estão muito próximas e, considerando a barra de variação, o comportamento é muito semelhante. Assim, nesta região não existe a contribuição acentuada de dado grupo funcional que possa evidenciar uma dependência com a idade.

A amida I apresenta modo vibracional centrado em $1637 \mathrm{~cm}^{-1}$ e está relacionada ao colágeno (Movasaghi et al., 2007). Na Figura 8 observa-se que o grupo $\mathrm{C}$ apresentou menor porcentagem no 
valor da área atribuída a esta biomolécula relacionando este comportamento à menor concentração de colágeno em indivíduos com maior idade. De fato, na epiderme envelhecida ocorre o achatamento da junção dermo-epidérmica com diminuição das fibras de ancoragem, especialmente de colágeno (Boisnic e Blanchet, 2005; Silva e Carneiro, 2001; Tzaphlidou, 2004) o qual degrada com o tempo, levando ao aumento das ligações cruzadas e desorganização das fibras (Hadshiew et al., 2000).

\section{Conclusão}

Os resultados obtidos mostraram a possibilidade de utilizar a espectroscopia Raman Confocal no entendimento do processo de envelhecimento em relação às biomoléculas. Os resultados das comparações intergrupos mostraram que o grupo de maior idade teve prevalência da banda da tirosina, mas um decréscimo da banda centrada em $875 \mathrm{~cm}^{-1}$ do ácido pirrolidônico. A banda da amida I, centrada em $1637 \mathrm{~cm}^{-1}$, atribuída à presença de colágeno, como também outras proteínas e lipídeos, apresentou uma menor quantidade no grupo $\mathrm{C}$, que poderia ser justificado pelo decréscimo na concentração de colágeno em função da idade. No entanto, a complexidade do espectro Raman da pele mostrou que a correlação entre os espectros Raman dos aminoácidos puros não é diretamente obtida.

\section{Agradecimentos}

Ao CNPq e a FAPESP pelo apoio financeiro.

\section{Referências}

Berry N, Charmeil C, Goujon C, Silvy A, Girard P, Corcuff P, Montastier C. A clinical, biometrological and structural study of xerotic skin. International Journal of Cosmetic Science. 1999; 1(21):241-52. PMid:18503442. http://dx.doi. org/10.1046/j.1467-2494.1999.196570.x

Boisnic S, Blanchet MC. Vieillissement cutané chronologique. Cutaneous chronologic aging. EMC Dermatologie Cosmetologie. 2005; 4(2):232-41. http://dx.doi.org/10.1016/j. emcdc.2005.10.002

Carvalho CS, Martin AA, Santo AME, Andrade LEC, Pinheiro MM, Cardoso MAG, Raniero L. A rheumatoid arthritis study using Raman spectroscopy. Theoretical Chemistry Accounts. 2011; 130:1211-20. http://dx.doi. org/10.1007/s00214-011-0905-0

Caspers PJ, Lucassen GW, Wolthuis R, Bruining HA, Puppels GJ. In vitro and in vivo Raman spectroscopy of human skin. Bioespectroscopy. 1998; 4:31-9. http://dx.doi.org/10.1002/ (SICI)1520-6343(1998)4:5+<S31:AID-BSPY4>3.0.CO;2-M

Caspers PJ, Lucassen GW, Puppels GJ. Combined in vivo Confocal Raman spectroscopy and Confocal Microscopy of human skin. Biophysical Journal. 2003; 85:572-80. http:// dx.doi.org/10.1016/S0006-3495(03)74501-9

Choi JH, Cho M. Calculations of intermode coupling constants and simulations of amide I, II, III vibrational spectra of dipeptides. Chemical Physics. 2009; 361:168-75. http://dx.doi.org/10.1016/j.chemphys.2009.05.016

Egawa M, Tagami H. Comparison of the depth profiles of water and water-binding substances in the stratum corneum determined in vivo by Raman spectroscopy between the cheek and volar forearm skin: effects of age, seasonal changes and artificial forced hydration. British Journal of Dermatology. 2007; 158(2):251-60. Epub 2007 Nov 28. PMid:18047517. http://dx.doi.org/10.1111/ j.1365-2133.2007.08311.x

Engelke M, Jensen JM, Ekanayake-Mudiyanselage S, Proksch E. Effects of xerosis and ageing on epidermal proliferation and differentiation. British Journal of Dermatology. 1997; 137:219-25. PMid:9292070. http:// dx.doi.org/10.1046/j.1365-2133.1997.18091892.x

Guirro E, Guirro R. Fisioterapia Dermato-Funcional. 3. ed. Rio de Janeiro: Manole; 2002.

Hadshiew IM, Eller MS, Gilchrest BA. Skin aging and photoaging: the role of DNA damage and repair. American Journal of Contact Dermatitis. 2000; 11(1):19-25. http:// dx.doi.org/10.1016/S1046-199X(00)90028-9

Harding CR, Long S, Richardson J, Rogers J, Zhang $\mathrm{Z}$, Bush A, Rawlings AV. The cornified cell envelope: an important marker of stratum corneum maturation in healthy and dry skin. International Journal of Cosmetic Science. 2003; 25(4):157-67. PMid:18494897. http://dx.doi. org/10.1046/j.1467-2494.2003.00175.x

Harding CR, Watkinson A, Rawlings AV, Scott IR. Dry skin, moisturization and corneodesmolysis. International Journal of Cosmetics Science. 2000; 22:21-52. PMid:18503460. http://dx.doi.org/10.1046/j.1467-2494.2000.00001.x

Lademann J, Caspers PJ, Van der Pol A, Richter H, Patzelt A, Zastrow L, Darvin M, Sterry W, Fluhr JW. In vivo Raman spectroscopy detects increased epidermal antioxidative potential with topically applied carotenoids. Laser Physics Letters. 2009; 1(6):76-9. http://dx.doi. org/10.1002/lapl.200810092

Laube S. Skin infections and ageing. Ageing Research Reviews. 2004; 3:69-89. PMid:15163103. http://dx.doi. org/10.1016/j.arr.2003.08.003

Movasaghi Z, Rehman S, Rehman IU. Raman spectroscopy of biological tissues. Applied Spectroscopy Reviews. 2007; 42(5):493-541. http://dx.doi. org/10.1080/05704920701551530

Nelson LD, Cox MM. Lehninger principles of biochemistry. 5th ed. New York: W.H. Freeman and Company; 2008.

Nicoletti MA, Orsine EMA, Duarte ACN, Buono GA. Hipercromias: Aspectos gerais e uso de despigmentantes cutâneos. Cosmetics \& Toiletries. 2002; 3(14):46-51.

Oliveira LJ, Rocha IM, Guillo LA. Ensaio radiométrico de tirosinase. Revista Brasileira de Farmácia. 2004; 85(1):5-6. 
Penteado SC, Fogazza BP, Carvalho CS, Arisawa EA, Martins AM, Martin AA, Martinho HS. Diagnosis of degenerative lesions of supraspinatus rotator cuff tendons by Fourier Transform-Raman spectroscopy. Journal of Biomedical Optics. 2008; 13(1):014018. PMid:18315376. http://dx.doi. org/10.1117/1.2841017

Phillips CL, Combs SB, Pinnell SR. Effects of Ascorbic acid on proliferation a collagen synthesis in relation to the donor age of human dermal fibroblasts. The Journal of Investigative Dermatology. 1994; 103(2):228-32. PMid:7518857. http:// dx.doi.org/10.1111/1523-1747.ep12393187

Raniero L, Canevari RA, Ramalho LNZ, Ramalho,Santos EAP, Bitar RA, Jalkanen KJ, Martinho HS, Martin AA. In and ex vivo breast disease study by Raman spectroscopy. Theoretical Chemistry Accounts: Theory, Computation, and Modeling (Theoretica Chimica Acta). 2011; 130(4-6):1239-47. http://dx.doi.org/10.1007/s00214-011-1027-4
Silva MR, Carneiro SCS. Cosmetics for the elderly. Clinics in Dermatology. 2001; 19:413-23. http://dx.doi.org/10.1016/ S0738-081X(01)00181-X

Tfayli A, Piot O, Pitre F, Manfait M. Follow-up of drug permeation through excised human skin with confocal Raman microespectroscopy. Journal Eur Biophys 2007; 36(8):1049-58. Epub 2007 Jun 13. http:// dx.doi.org/10.1007/s00249-007-0191-x 2007.

Tzaphlidou M. The role of collagen and elastin in aged skin: an image processing approach. Micron. 2004; 35:173-7. Pmid:15036271. http://dx.doi.org/10.1016/j. micron.2003.11.003

Walters KA, Roberts MS. Dermatologic, cosmeceutic, and cosmetic development: Therapeutic and novel approaches. Oxford: Taylor and Francis; 2008.

\footnotetext{
Autores

Patrícia Karen Oliveira, Maira Gaspar Tosato, Rani de Souza Alves, Airton Abrahão Martin*, Priscila Pereira Fávero, Leandro Raniero

Laboratório de Espectroscopia Vibracional Biomédica, Instituto de Pesquisa e Desenvolvimento - IP\&D,

Universidade do Vale do Paraíba - UniVap, Av. Shishima Hifumi, 2911, CEP 12244-000,

São José dos Campos, São Paulo, Brasil.
} 